\title{
LA-UR-19-22675
}

Approved for public release; distribution is unlimited.

Title: The Peculiar Acoustics of Rocks

Author(s): $\quad$ Ten Cate, James A.

Remillieux, Marcel

Intended for: $\quad$ Acoustics Today, magazine, feature article

Issued: $\quad$ 2019-06-20 (rev.1) 
Disclaimer:

Los Alamos National Laboratory, an affirmative action/equal opportunity employer, is operated by Triad National Security, LLC for the National Nuclear Security Administration of U.S. Department of Energy under contract 89233218CNA000001. By approving this article, the publisher recognizes that the U.S. Government retains nonexclusive, royalty-free license to publish or reproduce the published form of this contribution, or to allow others to do so, for U.S. Government purposes. Los Alamos National Laboratory requests that the publisher identify this article as work performed under the auspices of the U.S. Department of Energy. Los Alamos National Laboratory strongly supports academic freedom and a researcher's right to publish; as an institution, however, the Laboratory does not endorse the viewpoint of a publication or guarantee its technical correctness. 


\section{The Peculiar Acoustics of Rocks}

James A. TenCate

Address:

Geophysics Group

Earth and Environmental

Sciences Division

Mail Stop D446

Los Alamos National Laboratory

Los Alamos, New Mexico 87545

USA

Email:

tencate@lanl.gov

Marcel Remillieux

Address:

Geophysics Group

Earth and Environmental

Sciences Division

Mail Stop D446

Los Alamos National Laboratory

Los Alamos, New Mexico 87545

USA

Email:

mcr1@lanl.gov
Surprisingly, understanding how sound behaves in rocks is not very well-known.

\section{Introduction}

For centuries, humans have used rocks as building materials. From the Parthenon of ancient Greece (Pentelic marble; bit.ly/2THk7XL) to the more recent Strasbourg cathedral (Vosges sandstone; bit.ly/2TtjZLY) to the Texas State capital building (Texas pink granite; bit.ly/2Ts6wE8), rock endures.

Although the mechanical and acoustical properties of rocks should be very wellknown, it turns out that quite the opposite is true. Rocks, in fact, exhibit some very peculiar behaviors. In addition to often being nonlinear, they exhibit hysteresis when pushed and pulled and have mechanical properties that slowly vary in time. All of these behaviors may also vary in their response, depending on the stimuli. Thus, although in some ways rocks can be treated as simple solids, in other ways they behave more like fluids. The real answer, however, is somewhere in between.

Because of the complexity of the behavior of rocks, our quest is for measurements and for the simplest models that can capture all the important features of the peculiar behavior of a rock. Ultimately, such models can be used to predict and better understand what goes on beneath our feet.

\section{Motivation}

Why is wave propagation and, in general, the acoustics of rocks of interest? One answer is for building restoration, a research area of continued study. Exadaktylos et al. (2001) and many others, for example, describe the (nonlinear) mechanical and acoustical testing on the Pentelic marble of the Parthenon.

However, there are other more compelling (financial) motivations. Knowing the sound speed profiles and how waves propagate in the ground underneath can inform seismic prospecting, such as where and how deep to drill for oil and gas, or for carbon sequestration. For example, in 1935, Conrad Schlumberger was issued a US patent (No. 2,191,119; bit.ly/2T3raFU) for the use of a sonic logging tool for oil and gas research. Imagine trying to determine the depth of a highly reflective layer (which may have an oil reservoir trapped underneath) without knowing the sound speed profile of the subsurface rocks. A considerable amount of research and development by various oil companies over the years followed from that first patent.

Rock acoustics is also an invaluable tool in understanding our heavenly neighbors. For example, in the 1970s, there was additional interest in the acoustics of rocks as a result of the return of the Apollo moon rocks. Acoustics was used to study these rocks to help discern the history and geology of the Moon. More recently, the Mars Rovers (Spirit, Opportunity, Curiosity, and now InSight) all had or have instruments onboard to try and understand the rocks on Mars. The purpose of these instruments is to see if Marian rocks may have formed in a wetter environment. InSight has actually placed a seismometer on the surface of Mars to listen for marsquakes to 


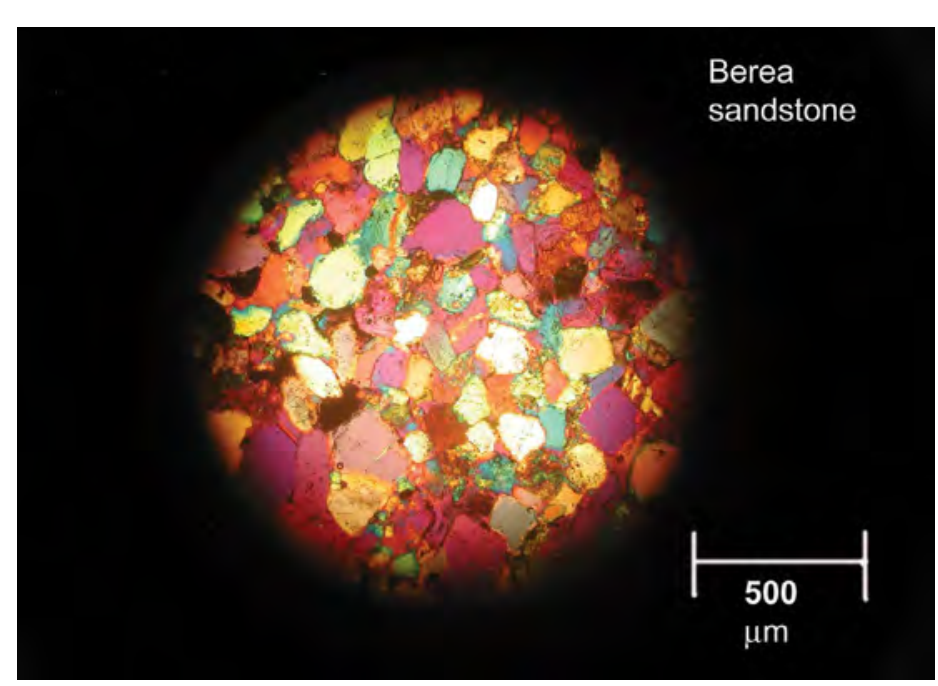

Figure 1. Thin section of a Berea sandstone sample under crosspolarizing filters. Different colors highlight different grain orientations. Black areas are voids, and some of the pore space between grains contains clays and silt in this sample. See text for further details.

aid and interpret the underlying structure beneath the surface (go.nasa.gov/2Fe9KRP; Smrekar et al., 2019).

Finally, the question of where to store high-level radioactive waste may find an answer in rocks too, such as in the Waste Isolation Pilot Plant (WIPP; wipp.energy.gov), a deep geological repository in New Mexico. For the WIPP, understanding rock properties (e.g., fluid and gas migration in the salt, how the rock was formed, mechanical behavior) in this case, and really in all cases above, is key.

\section{Properties of Rocks}

To better understand the mechanical and acoustical properties of rocks, it is instructive first to know something about how they are formed. Most of the rocks of financial interest are sedimentary. Indeed, sedimentary rocks are a focus of commercial oil and gas companies, The Office of Basic Energy Sciences, US Department of Energy, supports such research as well.

The primary building blocks of sandstones are weathered quartz grains that are cemented together under millions of years of pressure and fluid flow. These cementation processes are called lithification and/or diagenesis, and books are written about these processes and especially about those sandstones that are relevant to oil and gas exploration (Burley and Worden, 2009). For example, Berea sandstone (rocks are usually named for the places where they are first commonly found; Berea is a town in Ohio) is often used for research as a representative oil- and gas-bearing rock. It was formed about 400 million years ago from river sands deposited in what is now the US Midwest from an ancient river delta up in what is now Canada. It is perhaps worth noting that some buildings are constructed of Berea sandstone (e.g., the Johnson County Courthouse in Iowa; Rossman, 1975). What is especially notable about this sandstone is that the constituent grains are cemented together in such a way that they also contain extensive pore space. These pore spaces contain remaining fluids, occasionally some fine clays and silts, and in an oil and gas reservoir the remains of plants and animals in the form of oil.

Figure 1 shows a thin section of Berea sandstone, with the light through it observed through cross polarizers to best show off the crystalline orientation of the grains. The dark material in between the grains is the amorphous quartz and clays holding the rock together. From a mechanical and acoustical point of view, this imperfect grain-to-grain bonding, with distributions of stress concentrations via various grains and the ability for grains to rotate in and out of pore spaces under stress, make for an interesting solid.

How does a stress change or acoustic pulse get transmitted through such a collection of grains? Unlike a homogeneous solid, forces are not distributed smoothly through a rock. Each individual grain is cemented to its neighbors in various random ways. The particular collection of grains that participate, and not all do, in transferring a force or acoustic wave through a sandstone is called a force chain. Many hundreds of these force chains might participate in wave propagation or maybe just a few. You can imagine that building a (finiteelement) model of such a rock is no easy task. Add trapped fluids to that matrix, and you have a recipe for fascinating and challenging material behavior.

\section{Ideal Solid Versus the Behavior of a Rock}

So how does such an interesting solid behave? The mechanical quasi-static behavior will be examined first. Imagine first a spring that obeys Hooke's law, $F=-k x$, where $F$ is the required force to extend or compress a spring, $k$ is the spring constant, and $x$ is the distance to be moved. (Of course, such a spring cannot be compressed too much lest the coils of the spring will come in contact with each other.) Put the spring in a stress-strain (load-frame) machine, compress and release 


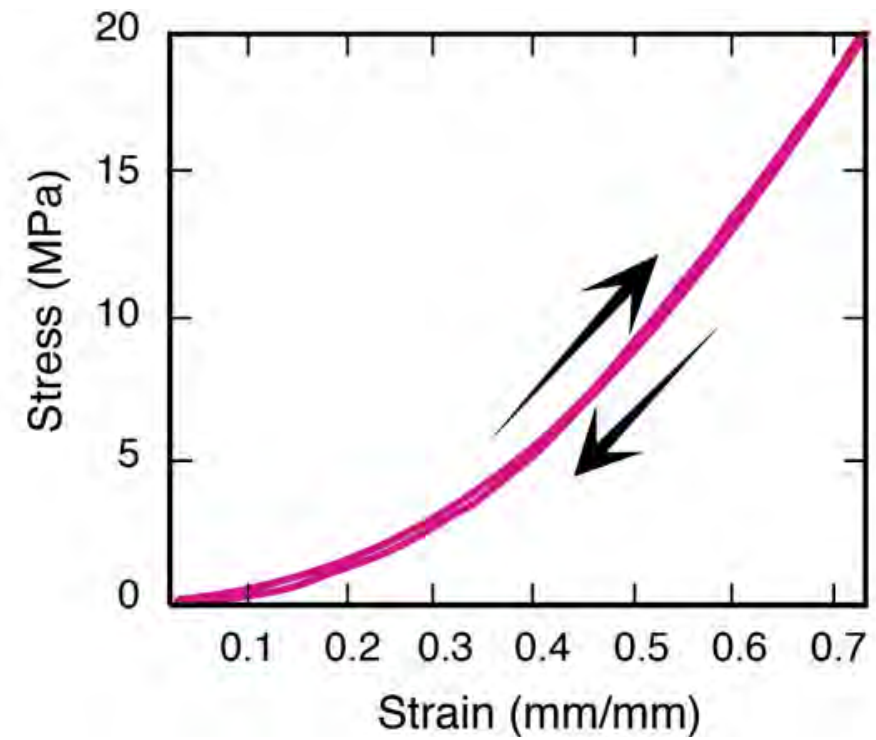

Figure 2. Very slow stress-strain measurement on a sample of Berea sandstone (over four days). Stress is applied linearly and then released at the same rate. The error bars in the strain measurements (not shown for clarity) are \pm 0.02 . Upward and downward curves are the same within the error bars. (Plot axes are swapped because of tradition; strain always goes on the $\mathrm{y}$-axis.)

it, and plot the resulting stress-strain data. The experiment results in a straight-line force-displacement curve.

Many solids also have straight-line stress-strain curves for small forces. In the solid, there is an additional Poisson effect, it bulges as it is squeezed, that can usually be ignored in a rock. Now put a small core sample of Berea sandstone in that stress-strain (load-frame) machine, compress and release it, and plot the resulting stress-strain data. As seen in Figure 2, Berea sandstone does not behave like a linear spring or solid. Instead, the rock gets stiffer as it is compressed.

This is not surprising considering the structure of the rock. Much like squeezing a linear spring so hard that the coils touch, the applied force is squeezing grains into the pore spaces, and eventually, they all come in contact with one another. This behavior is not new or surprising. In fact, it has been known since the early twentieth century when Adams and Coker (1906) published a series of quasi-static nonlinear stress-strain curves for a wide variety of rocks and showed that many were nonlinear. You might note that these measurements are all in large strains and are quasi-static (i.e., at extremely low frequencies). How is this relevant to acoustics where the effective strains and frequencies are quite different? As we discuss in Wave Propagation, nonlinearity can be seen in measured waveforms too. So, is it sufficient to just add nonlinearity to a rock to describe its behavior? No.

There are two additional and important complications to the stress-strain behavior inherent in a rock. The stress-strain curve in Figure 2 was taken at a very slow rate (data were acquired over a period of several days). The nonlinearity is clearly present, and the compression phase essentially matches the release phase (within errors). However, the rate at which the stress-strain data are acquired matters. Imagine repeating the stress-strain measurement in Figure 2, this time linearly, increasing and then decreasing the stress at different rates and measuring the resultant strain. The results of taking data slowly (e.g., days) and then quite quickly (e.g., minutes) on a pair of different sedimentary rocks is surprising.

Figure 3 shows four sets of stress-strain measurements on two sedimentary rocks, two done over the period of a several days and two done at normal laboratory timescales of less than an hour (see Claytor et al., 2009). The fast measurements (Figure 3, right) both start to take on distinctive banana shapes. These are, in fact, hysteresis loops similar to what is seen in ferromagnets. But the fact that different hysteresis loops are measured depending on the rate at which the experiment is
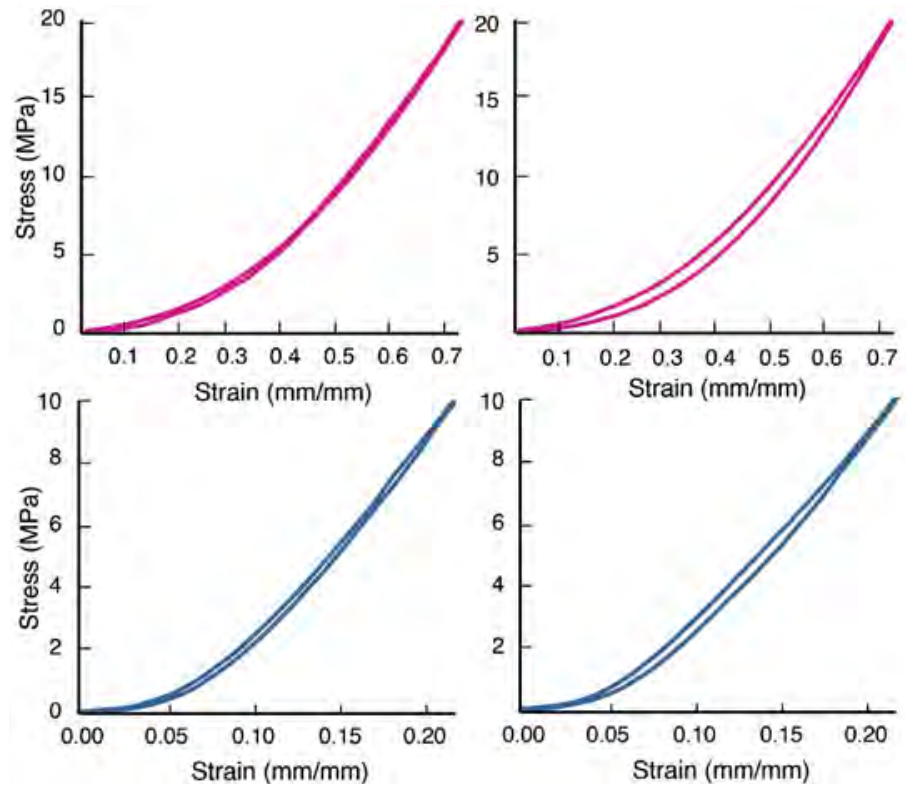

Figure 3. Stress-strain plots of two sedimentary rock samples: Fontainebleau sandstone (top, red) and Berea sandstone (bottom, blue). Left plots were made at a very slow rate (days); right plots were made at a reasonably fast rate (30 minutes). Hysteresis, manifested as banana-shaped loops, is apparent in the fast measurements (right). Measurement rate matters. See text for further discussion. 


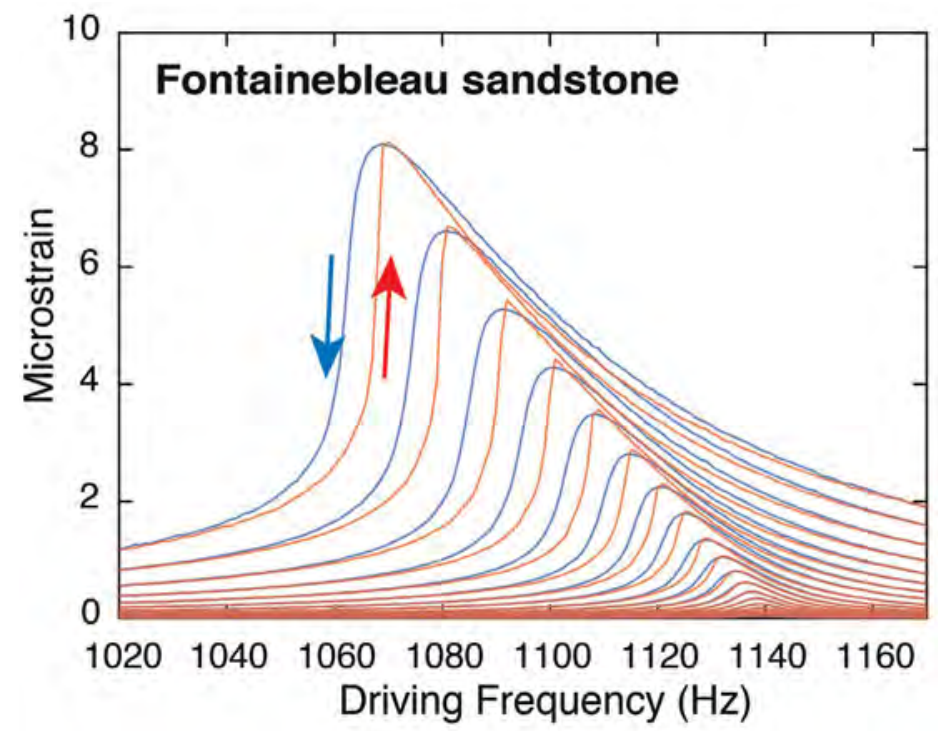

Figure 4. A series of resonance curves at increasing amplitudes for the lowest resonance frequency of a long thin bar of Fontainebleau sandstone. The driving frequency is swept upward (red) and downward (blue), and the response of the sample is measured with a laser vibrometer and converted to strain. Note the softening nonlinearity (resonance frequency drops) with increasing excitation amplitude and the different up-and-down curves that result from slow dynamics.

done complicates the behavior of a rock much more. A rock is not only nonlinear; there is hysteresis and there is some kind of time-dependent behavior present as well.

The measurements described above are quasi-static and at fairly large stresses and strains. What about at lower strains and higher frequencies? Does hysteresis still persist, can it be measured, and does it really matter for acoustics? Lest you think this hysteretic behavior isn't present in wave propagation, it is. At much lower strains and at higher frequencies, those typical for seismic waves, you see "cuspy" triangular waveforms, also indicating the presence of hysteresis (McKavanagh and Stacey, 1974). Some of these waveforms are seen at even higher frequencies and are shown in Wave Propagation. Hysteresis is present at many frequencies and amplitudes of interest to the acoustician.

Perhaps it should not be surprising that the hysteresis described above and seen in Figure 3 was also found to be time dependent. There is a term for this behavior, elastic aftereffect (e.g., see Becker, 1925), and it has an analogy with magnetic materials where the effect takes place over years instead of days. Elastic aftereffect is present in rocks, and it fortunately (or unfortunately) manifests itself on timescales relevant to most acoustic measurements. To clarify discussions about rocks, the rate-dependent effects were dubbed "slow dynamics" in the geophysics literature quite a while ago and the name stuck (TenCate and Shankland, 1996).

For the acoustician then, questions remain. Are nonlinear, hysteretic, and slow-dynamic effects large in rocks? Can they be seen in wave propagation down a long thin bar or maybe in the acoustic waves sent across a block of sandstone? What about nonlinear wave interactions in rocks; do they occur too? These kinds of questions have been the focus of roughly 25 years of research of a group of researchers and colleagues at the Los Alamos National Laboratory and many others elsewhere. The acoustical experiments to date fall into two distinct types.

\section{Two Kinds of Experiments}

\section{Resonance}

The first type of experiment, resonance measurements on long thin bars, is occasionally used to characterize the mechanical properties of rock cores. Long thin cores have geometries that make it easy to find and precisely locate resonance frequencies. These are useful to characterize the elastic moduli and intrinsic attenuation of the core.

However, with increasing excitation amplitudes, something surprising happens. A series of resonance curves taken around the lowest resonance frequency, 1-D wave motion that in a solid resembles a snake swallowing, takes on peculiar shapes with increasing drive amplitude. Figure 4 shows a family of up-and-down sweeps of frequency versus amplitude for yet another sedimentary rock, this one from Fontainebleau, south of Paris, France. The resonance frequency drops, and the upward and downward curves are not the same. These curves resemble those of softening nonlinear spring-mass systems, such as those whose motion is described by a Duffing equation. The springs in a Duffing equation do not obey (linear) Hooke's law and have a cubic nonlinear spring constant. Upward and downward sweeps in frequency (versus amplitude) produce different resonance curves. Even the jump in the upward sweeps resembles a Duffing oscillator spring-mass system. In fact, a great number of early studies went into trying to fit the resonance curves of rocks with the Duffing models. However, although a nonlinear spring-mass description works quite well down at very low strains, where nonlinearity dominates, above a certain drive level, slow dynamics matters more. 


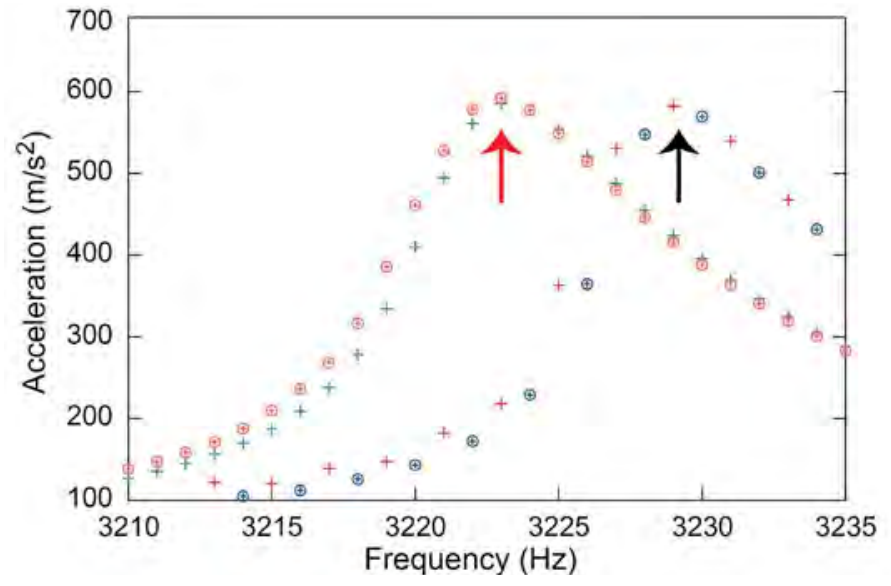

Figure 5. Up-and-down resonance curve data points taken quickly (2 minutes; orange circles, upward data points; green plus symbols, downward data points) and taken very slowly (2 days; blue circles, upward data points; red plus symbols, downward data points), both at the same excitation amplitude. In contrast with the smooth curves shown in Figure 6, resonance curve data were taken sparsely, point by point. Error in measurement is $\pm 20 \mathrm{~m} / \mathrm{s}^{2}$. Red arrow, resonance frequency for the slow experiment at 3,239 Hz; black arrow, resonance frequency for the fast experiment at 3,223 Hz, a significant difference.

Examples of the above behavior abound. Depending on how fast or slow the resonance sweep is done, the slope, jumps, and shapes of all the resonance curves can vary dramatically (TenCate and Shankland, 1996). In particular, by doing the sweep measurements very slowly or very quickly, it was possible to get two very different resonance curves from the same rock sample (see Figure 5). Rate is the only variable here. In fact, later work shows that the resonance behaviors at frequencies and amplitudes of most interest to the acoustician are almost entirely dominated by slow-dynamic rate effects (Pasqualini et al., 2007; Remillieux et al., 2017). Classical nonlinearity of the sort that an acoustician would expect (e.g., which cause sonic booms), although large, takes a back seat to slow dynamics in many cases.

\section{Wave Propagation}

Wave propagation experiments fall into the second type of measurement carried out by Los Alamos researchers and colleagues. Are nonlinear, hysteresis, and rate effects visible in these kinds of experiments too? Yes indeed, they are all present. Numerous papers show nonlinearity in the form of harmonics and at strains roughly 1,000 times less than the typical quasi-static stress strain measurements (e.g., Meegan et al., 1993; TenCate et al., 1996; Remillieux et al., 2017). Recall that McKavanaugh and Stacey (1974) show that cuspy hysteresis is present in wave propagation measurements as well as in rate effects.

Remillieux et al. (2017) showed such behavior at higher frequencies and lower strains. Figure 6 shows the results of sending a tone burst down a long thin Berea sandstone bar. The waveforms are recorded 0.5 meter from the source and at several different increasing source amplitudes. Figure 6, inset, shows just how distorted the waveform has become. The triangulation is severe, and even the zero crossings have moved. Further in-depth discussion and more results can be found in the aforementioned paper.

What sort of physics is causing the peculiar behavior of rocks? Recall that a sandstone typically consists of a random collection of quartz grains cemented together in unusual ways, with odd contact surfaces and porosity for the grains to move and rotate around under stress. In addition, a rock may also have fluids trapped within that matrix during its formation. These fluids may also play a role, especially at crack tips and contact points. Bittner and Popovics (2019) made a remarkable observation under an environmental scanning electron microscope (a scanning electron microscope that can visualize wet objects) that shows fluids in a cement pore disappearing under acoustic excitation and reappearing after the acoustic excitation is turned off. A video of the experiment shows an initially wet pore (youtu.be/H_ahCbbYt3Q). However, when an acoustic field is turned on, all the water disappears. Once the acoustic field is turned off, water is seen seeping back into the pore.

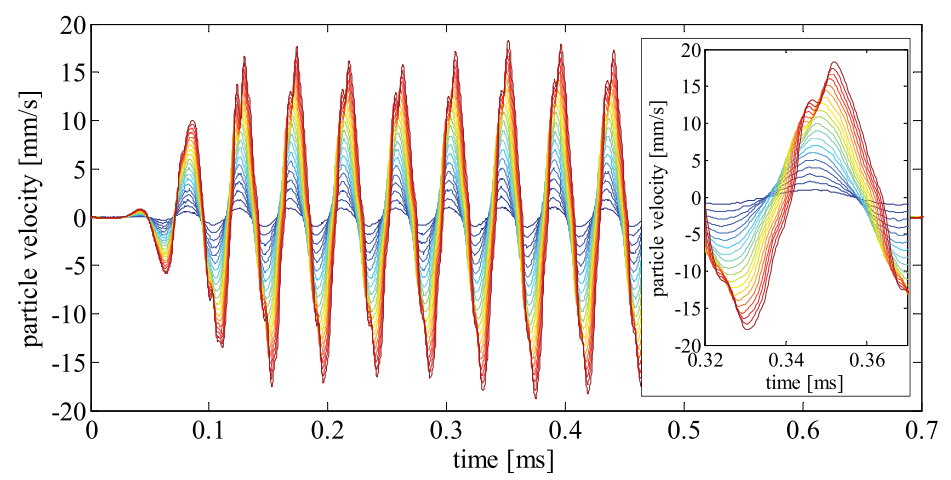

Figure 6. Distorted waveforms measured 0.5 meter from the source, a tone burst, sent down a long thin Berea sandstone bar at increasing drive amplitudes (blue to red). Inset: zoom of one cycle and shows how distorted these waveforms become. The spectrum of these waveforms is dominated by odd harmonics. The waveforms also show how the pulse is progressively delayed with increasing amplitudes at the zero crossings. There is more than nonlinearity present in these waveforms. 
It has also been suggested by Page et al. (2004) and many others that there may be disturbances within the quartz crystals themselves. Perhaps nonlinearity is a result of the movement of crystalline lattice structures (i.e., dislocations) under an acoustic excitation. However, such physics at the crystalline level do not appear to play a role. A set of experiments where quasi-static stress-strain curves were obtained simultaneously with neutron-scattering measurements (Darling et al., 2004) shows that the average crystalline behavior in a sandstone is linear (i.e., each grain behaves as a perfect little spring), even though the macroscopic stress-strain curves are quite nonlinear! It seems reasonable to conclude that it is the geometric structure of the entire cemented-grain matrix that is largely responsible for the nonlinearity and not the crystals themselves. In addition, having fluids present at the contact surfaces seems to be necessary as well. Researchers are actively seeking other experiments to learn more about the processes at the grain scale and larger levels responsible for the behavior seen in rocks.

\section{Conclusions}

In summary, there are three general classes of behavior that have been observed in rocks: (1) classical nonlinearity; (2) hysteresis; and (3) slow dynamics. There are also three important features in rocks that we think are the key contributors to the observed behavior: (1) grain-to-grain contact dynamics; (2) geometrical arrangement of grains (e.g., how they are cemented and oriented and how much pore space there is between them); and (3) fluids that provide lubrication between grains, fluid-grain surface tension effects, and maybe even high-pressure chemistry at contact points.

Many key questions about the acoustic behavior of rocks still remain to be answered. What is the connection between the proposed physics described in this article and the observed behavior? To further understanding, there is a great need for more very small scale experiments, like the one done by Bittner and Popovics (2019). There is also a need for very detailed finite-element models with all the above physics embedded, as now being done at Los Alamos National Laboratory, and perhaps also working with larger than life-scale representative models. The quest is for the simplest models that capture all the important features of the peculiar behavior of a rock. Ultimately, such models can be used to predict and better understand what goes on in the earth deep beneath us.

\section{Acknowledgments}

We gratefully acknowledge the sustained and continuous support from the Office of Basic Energy Sciences, US Department of Energy.

\section{References}

Adams, F. D., and Coker, E. G. (1906). An Investigation into the Elastic Constants of Rocks, More Especially with Reference to Cubic Compressibility. Publication No. 46, Carnegie Institution of Washington, Washington, DC.

Becker, R. (1925). Elastic aftereffect and plasticity. Zeitschrift für Physik 3, 185-213. https://doi.org/10.1007/BF01328304

Bittner, J. A., and Popovics, J. S. (2019). Direct imaging of moisture effects during slow dynamic nonlinearity. Applied Physics Letters 114(2), 021901. https://doi.org/10.1063/1.5063904

Burley, S., and Worden, R. (Eds.). (2009). Sandstone Diagenesis: Recent and Ancient. John Wiley \& Sons, New York.

Claytor, K. E., Koby, J. R., and TenCate, J. A. (2009). Limitations of Preisach Theory: Elastic aftereffect, congruence, and end point memory. Geophysical Research Letters 36(6). https://doi.org/10.1029/2008GL036978

Darling, T. W., TenCate, J. A., Brown, D. W., Clausen, B., and Vogel, S. C. (2004). Neutron diffraction study of the contribution of grain contacts to nonlinear stress-strain behavior. Geophysical Research Letters 31(16). https://doi.org/10.1029/2004GL020463

Exadaktylos, G. E., Vardoulakis, I., and Kourkoulis, S. K. (2001). Influence of nonlinearity and double elasticity on flexure of rock beams - II. Characterization of Dionysos marble. International Journal of Solids and Structures 38(22-23), 4119-4145. https://doi.org/10.1016/S0020-7683(00)00252-3

McKavanagh, B., and Stacey, F. D. (1974). Mechanical hysteresis in rocks at low strain amplitudes and seismic frequencies. Physics of the Earth and Planetary Interiors 8(3), 246-250. https://doi.org/10.1016/0031-9201(74)90091-0

Meegan, G. D., Jr., Johnson, P. A., Guyer, R. A., and McCall, K. R. (1993). Observations of nonlinear elastic wave behavior in sandstone. The Journal of the Acoustical Society of America 94(6), 3387-3391. https://doi.org/10.1121/1.407191

Page, K. L., Proffen, T., McLain, S. E., Darling, T. W., and TenCate, J. A. (2004). Local atomic structure of Fontainebleau sandstone: Evidence for an amorphous phase? Geophysical Research Letters 31(24). https://doi.org/10.1029/2004GL021717

Pasqualini, D., Heitmann, K., TenCate, J. A., Habib, S., Higdon, D., and Johnson, P. A. (2007). Nonequilibrium and nonlinear dynamics in Berea and Fontainebleau sandstones: Low-strain regime. Journal of Geophysical Research: Solid Earth 112(B1). http://doi.org/10.1029/2006JB004264

Remillieux, M. C., Ulrich, T. J., Goodman, H. E., and TenCate, J. A. (2017). Propagation of a finite-amplitude elastic pulse in a bar of Berea sandstone: A detailed look at the mechanisms of classical nonlinearity, hysteresis, and nonequilibrium dynamics. Journal of Geophysical Research: Solid Earth 122(11), 8892-8909. http://doi.org/10.1002/2017JB014258

Rossmann, A. J. (1975). National Register of Historic Places Inventory/Nomination: Johnson County Courthouse. National Park Service, Washington, DC.

Smrekar, S. E., Lognonné, P., Spohn, T., Banerdt, W. B., Breuer, D., Christensen, U., Dehant, V., Drilleau, M., Folkner, W., Fuji, N., and Garcia, R. F. (2019). Pre-mission InSights on the interior of Mars. Space Science Reviews 215(1), 3. http://doi.org/10.1007/s11214-018-0563-9 
TenCate, J. A., and Shankland, T. J. (1996). Slow dynamics in the nonlinear elastic response of Berea sandstone. Geophysical Research Letters 23(21), 3019-3022. https://doi.org/10.1029/96GL02884

TenCate, J. A., Van Den Abeele, K. E., Shankland, T. J., and Johnson, P. A. (1996). Laboratory study of linear and nonlinear elastic pulse propagation in sandstone. The Journal of the Acoustical Society of America 100(3), 13831391. https://doi.org/10.1121/1.415985

\section{BioSketches}

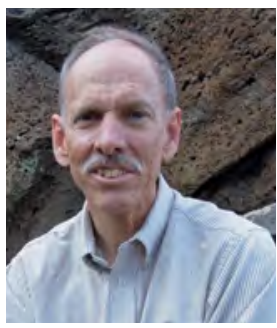

James TenCate started his acoustics career as a musician who quickly developed a keen interest in acoustics. After college (physics), he looked for graduate schools that specialized in acoustics and ended up at the University of Texas at Austin working under David Blackstock. During that time, he developed an appreciation and understanding of nonlinear acoustics in air and then in water. At the time, Los Alamos National Laboratory, Los Alamos, NM, was building a team to study nonlinearity in rocks. TenCate joined the Geophysics Group as a postdoc and spent the rest of his acoustics career at Los Alamos. TenCate is a Fellow of the Acoustical Society of America.

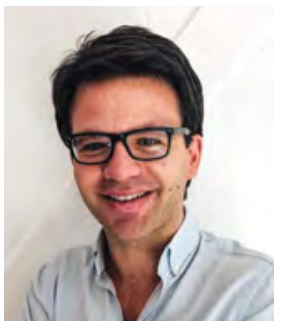

Marcel Remillieux has been a member of the Geophysics Group at Los Alamos National Laboratory, Los Alamos, NM, since 2013, first as postdoc and now as research scientist. He specializes in using nonlinear acoustics for nondestructive testing, from the integrity of structures in the nuclear energy industry to geophysical exploration for the oil and gas industry. His background is in acoustics, with MS and $\mathrm{PhD}$ degrees obtained from Virginia Tech, Blacksburg, VA, in Prof. Fuller's group. In his spare time, he and his wife Stephanie run Fleur de Lys, a busy and cozy French bakery/café in the heart of the atomic city.

\section{BE HEARD!}

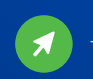

Students, get more involved with the ASA

through our student council at:

asastudentcouncil.org/get-involved

\section{NGC TESTING SERVICES ACOUSTICAL - FIRE - STRUCTURAL - ANALYTICAL}

Over 50 Years of Test Services

NVLAP Accredited (Code 200291-0)

IAS Accredited (Lab Code 216)

ISO/IEC 17025 Compliant

Test Capabilities Include:

E492 IIC, E2179 Delta IIC E90 STC C423 NRC, E1414 CAC, E1111 AC

Sound Power, Pipe Lagging, SAE J1400

E84 Flame Spread, E119 Fire Endurance

NGC Testing Services • 1650 Military Road

Buffalo, NY 14217 - Tel: 716-873-9750 ngctestingservices.com

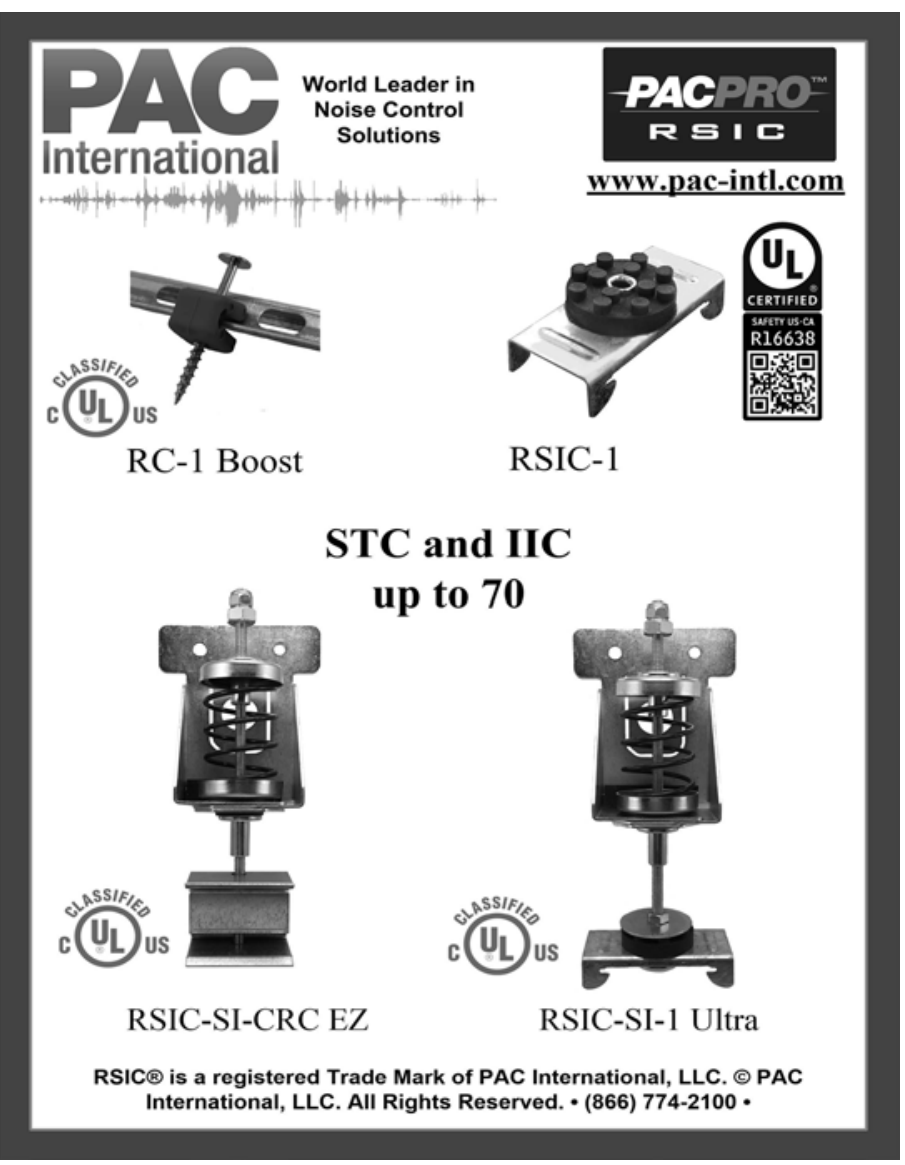

